\title{
DETERMINANTS OF VARIANCE IN COST METHOD OF VALUATION IN UYO, NIGERIA
}

\author{
Paul C. Anih \\ Department of Estate Management \\ University of Uyo in Nigeria \\ e-mail:paulanih@uniuyo.edu.ng

\section{Sunday Usenemana} \\ Department of Estate Management \\ University of Uyo in Nigeria \\ e-mail:usen163@yahoomail.com
}

\begin{abstract}
Variance in the cost method of valuation as caused by certain variables is objectionable in the profession of estate surveying and valuation at both local and international best practices. The study aimed at determining the variables for the variance in the application of the cost method of valuation for estate surveying and valuation practice in Uyo. The sample of the population used for the research comprised 110 estate surveyors in Uyo. The survey method was used for the research. The sampling technique was purposive sampling. The data were analyzed using standard deviation and analysis of variance (ANOVA). The findings showed that depreciation rates of $\sigma 2=113.974$ and $\sigma=10.676$ were over $60 \%$ of the total variance of values among estate surveyors and valuers. The construction rates and errors in manual measurements and computation of areas, volumes and dimensions showed $02=$ 6.004 and $\sigma=2.4503$, which accounted for $15 \%$ of total variance. The study recommends the use of a regional-based scientific approach to measure depreciation for consistency and high precision. Automated techniques, such as GPS, AutoCAD, Google Earth pro, are recommended to minimize variance in measurements.
\end{abstract}

Keywords: analysis of variance, capital value, cost method, valuation, variance.

JEL Classification: Q51, R20, R30, R39, R00.

Citation: Anih, P. C., \& Usenemana S. (2021). Determinants of variance in cost method of valuation in Uyo, Nigeria. Real Estate Management and Valuation, 29(3), 77-86.

DOI: https://doi.org/10.2478/remav-2021-0023

\section{Introduction}

This study identifies the variables responsible for variance in the cost method of valuation in order to resolve ways of sustaining accuracy in the valuation report. The Green Book (2019) approves that, in applying cost approach, the valuers must follow the stipulated guidelines contained in International Valuation Standard (IVS) 105 paragraph 70.1 - 14. This approach guides the application of valuation of real property interests using the depreciated replacement cost (DRC) method. DRC is used where there is no active market for the asset being valued - that is, where there is no useful or relevant evidence of recent sales transactions due to the specialized nature of the asset and where it is impractical to produce a reliable valuation using other methods (RICS Guidance Note, 2018; Green Book, 2019).

In Uyo, the cost method of valuation is the most frequently used method of valuation due to the valuation briefs (compulsory acquisition valuation) and the massive acquisition of landed properties in the oil-rich Niger Delta for public development projects. However, the Laws of the 
Federation of Nigeria (LFN) Cap L5, 2004 recommends payment of compensation to owners of properties compulsorily acquired for overriding public interest. Furthermore, the law regulating compulsory acquisition and payment of compensation which is contained in the Land Use Act approves that the depreciated replacement cost (DRC) method should be used for the valuation of the properties to be compulsorily acquired for overriding public interest. The choice of adopting the cost method of valuation in Uyo is based on legislative recommendations. In addition to this, the use of the depreciated cost method for valuation in the area was due to some conditions which other methods of valuation could not appropriately render. Observation has shown that some of the Estate Surveyors and valuers adopt variables of the depreciated replacement cost method, as they are familiar with the method and its application.

It is important to note that the cost method is not free of challenges, such as subjective judgments in depreciation, measurement errors and use of statutory rates (compensation rates) for capital value estimation, which contributes to discrepancies among Estate Surveyors and valuers in their opinion. The method, as used by some Estate Surveyors and valuers, could be laden with subjectivity in its application, impaired by the manual and subjective measurement approaches and non-use of objective methods of measuring the depreciation of building. Real property valuation is a valuable economic decision-making tool that requires extreme care. Hence, Kalu (2001) emphasized that a Valuer should follow a well-defined, systematic and, at times, scientific approach in arriving at an assessment of value. Jorge (2015) argued that valuation is an extremely important factor in maintaining a stable financial market climate and national economy as it is assuming a great role within the present global economic trend. Sometimes financial institutions hamper access to mortgages due to increased credit risk and outstanding claims, as well as regulations which require capitalization and increased core capital ratio of financial institutions.

\section{Literature Review - Valuation Variance in Cost Method}

Previous studies have concentrated on valuation variance in investment properties with inattentiveness to determinants of valuation variance in the cost method (Ayedun, et al., 2012; Effiong 2015). In the cost approach to valuation, valuers often collect and measure variables resulting in high levels of discrepancies among valuers (Adegoke, 2016). The essence of valuation is to determine the market value of properties by using objective and scientific methods with high precision in capital value determination (Effiong, 2015). Hence, the need to observe careful assessment is important especially when subjectivity, which might result in the variance of values, is applied (IVSC, 2019).

A particular method that is adopted most often for statutory and non-statutory valuation, especially for special properties, is the cost method of valuation. However, where there is no market evidence coupled with the passage of time, the depreciated replacement cost, which is an aspect of the cost method, is adopted (Yeboah \& Ayitey, 2006). Applying the cost method of valuation by real estate professionals revealed that a client's perception of the quality of the valuation report is needed for a high valuation standard. Effiong (2015) recalled how the situation in the United Kingdom and Australia attracted public criticism, which led to the damaging effect of public confidence in the valuation profession and valuation process. Hutchison et al. (1995) and Adair et al. (1996) carried out a study in the UK using hypothetical properties, which five national valuers and five local valuers used the same set of information on; both studies showed that valuation variance among the five National and five local valuers firms was $8.63 \%$ to $11.86 \%$ respectively. The study also showed that $80 \%$ of valuation had a mean deviation of less than $20 \%$ as observed with a wider variance in valuation. Hager and Lord carried out a foremost work on valuation accuracy. The study showed significant variance among 10 valuers on two properties, despite the use of similar data and the same method of valuation. Another notable case was the valuation of the Queens Oat Hotel chain by two firms of valuers in the UK, which received criticism of its valuation reports (Adair et al., 1996). A comparison by two valuers produced a difference of $\$ 120$ million or $16.6 \%$ (Parker, 1998).

Roca (1996) observed that the subjective parameters existing in the methods of valuation are the causes of inconsistency and variance in property values. He further observed that the replacement cost method's subjectivity is linked to the determination of depreciation charge. In the determination of market value for residential non-investment properties or special properties which requires the use of cost method, Jorge (2015) observed that the major area of subjectivity which results in inconsistency of value is intuitive reasoning. This is used by some estate surveyors and valuers to estimate the level of depreciation, which often differs considerably. This also results in disparity in the valuation opinion of 
estate surveyors and valuers carrying out valuation of the same property with the same data (Ayedun, 2012).

\subsection{Elements of Cost Method of Valuation}

Millington (2009) and International Valuation Standards (IVS) (2013) identified three main variables to be considered when carrying out DRC, which are site value, building value or the replacement cost (gross floor area multiplied by the rate of construction and depreciation). The IVS (2013) defines depreciated replacement cost (DRC) as a method of valuation which is based on the estimate of current market value of land for its existing use plus the current gross cost of improvements less allowances for physical deterioration and all relevant forms of obsolescence and optimization.

The building value is the cost of constructing a new building or the net replacement cost of the building, the reproduction cost is the cost of creating a virtual replica of the existing structure, employing the same design and similar building materials. However, the replacement cost is the estimated cost to construct, at current prices, a building with equivalent utility, using materials and standards, design and layout that are currently used in the market (Taquem \& Academy, 2016). The Taquem Academy adopted three methods for estimating the cost of buildings, which were: the comparative unit method, the unit-in-place method and the quantity surveyors method; Taquem Academy, on the other hand, noted that the comparative unit method is the simplest of the three methods and most widely used.

The process in this method includes; determination of the area of the building being assessed. This is not necessary if the building plan is available. Secondly, the cost per square meter needs to be ascertained. Thirdly, multiply the area of the subject property by appropriate rate to give a preliminary estimate of the current cost of the construction. Fourthly, make cost adjustments for features not common between the building being assessed and the model building from which the cost per square meter was derived and adjusted for depreciation. Buildings are known to age with passage of time. Consequently, the depreciated replacement cost, involves depreciation of the replacement cost using any of the linear and non-linear depreciation models (Yeboah and Ayitey, 2006). Bello (2014) and Bello et al. (2015) conducted studies in Lagos and observed that estate surveyors and valuers mostly use the rule of thumb method to estimate depreciation. However this method relies on subjective judgement and experience, and hence valuers' estimates vary based on their experience and sense of judgment.

\subsection{Regional Based Approach for Estimating Depreciation}

Plimmer and Sayce (2006) observed that, because there is no strict and definitive methodology, there are numerous scopes for valuers to change the use of their methods. Kalan (2018) reiterated the need for a regional specific depreciation formulae in highway capital stock to ensure a more precise and consistent estimate of depreciation. The Royal Institute of Chartered Surveyors (RICS) (2019) recognized the need for legislative requirements for regional, national or international approaches in application of the depreciated replacement cost method. Gorzig (2007) showed how methodological differences exist among European Union countries and recommended the application of more standardized methods by harmonization of the assets and a breakdown of the industry. A harmonised asset and industry breakdown will improve the possibilities to separate structural influence from methodological influence, which, he claims, centers on the harmonization of service life assumptions, determined by economic factors which differ within each region or country.

\section{Data and Method}

The population of the study was the practicing registered estate surveyors and valuers in Uyo, Nigeria. The total number of registered estate surveyors and valuers in Uyo was 110 (NIESV Directory, 2018). In view of the relatively small size of the population, sample size (110) was adopted for the study. A survey approach was used. The Instrument designed for the data collection were structured close-ended questionnaires. The data collected during the study were presented using relative frequency tables. The statistics used were analysis of variance $(S)$, standard deviation (SD) and CV (Coefficient of variation). The F ratio analysis was used to determine the level of significance of the existing variance in the coefficient of variation of the cost method elements among the Estate Surveyors and valuers. The formulas for the variance, standard deviation and Coefficient of variation applied in cost method were given as: 


$$
\text { Variance, } \sigma^{2}=\frac{\sum(X-x)^{2}}{N} \text { and Standard Deviation, } \sigma=\sqrt{ } \frac{\sum(X-x)^{2}}{N}
$$

where $\sigma^{2}=$ Population Variance

$\sigma=$ Population standard deviation

$\mathrm{x}_{1}=\mathrm{SV}=$ Site Value

$\mathrm{x}_{2}=$ GFA Gross Floor Area

$\mathrm{x}_{3}=\mathrm{RC}=$ Construction Rate $/ \mathrm{m}^{2}$

$\mathrm{x}_{4}=$ Dep. $=$ Depreciation Rates expressed in percentage or Naira $(\mathbb{N})$.

Coefficient of Variation (CV) is given by

$$
\frac{\text { Standard Deviation } \cdot 100}{\text { Arithmetic Mean }}=\frac{\sigma .100}{\mu}
$$

where $\sigma=$ Population standard deviation; $\mu=$ population mean

The F-ratio analysis; $\mathrm{F}=\frac{S_{1}^{2}}{S_{2}^{2}}$, where $S_{1}^{2}=\frac{\sum X_{1}-\overline{\bar{X}} \bar{X}_{1}}{n_{1}-1}$ and $S_{2}^{2}=\frac{\sum X_{2}-\overline{X_{2}}}{n_{2}-1}$. It important to note that $S_{1}^{2}$ is always a larger estimate than the $S_{2}^{2}$ i.e. $S_{1}^{2}>S_{2}^{2}$

$$
\begin{gathered}
\mathrm{F}=\frac{\text { Larger estimate of variance }}{\text { Smaller estimate of variance }} \\
V_{1}=n_{1}-1 \text { and } V_{2}=n_{2}-1
\end{gathered}
$$

$v_{1}=$ Degree of freedom for larger variance

$V_{2}=$ Degree of freedom for smaller variance

\section{Empirical Results}

\section{Table 1}

Distribution of Questionnaire

\begin{tabular}{cccc}
\hline S/N & Category & Frequency & $\%$ \\
\hline $\mathbf{1 .}$ & Returned questionnaires & 78 & 70.9 \\
\hline $\mathbf{2 .}$ & Not returned questionnaires & 32 & 29.1 \\
\hline & Total & $\mathbf{1 1 0}$ & $\mathbf{1 0 0}$ \\
\hline
\end{tabular}

Source: Authors' survey, 2020.

Table 1 shows the distribution of the questionnaires. Two items were observed under the categories of returned and not returned questionnaires: frequency and percentage. The first item is the returned questionnaires with a frequency of responses equal to 78 , represented by $70.9 \%$ of the population. The second category is unreturned questionnaires, with a frequency of 32, making for $29.1 \%$. The total of the questionnaires distributed was 110 , which represents $100 \%$ of the sample.

Table 2 shows respondents' years of experience. The respondents were classified into two groups: Associates and Fellows. Table 2 shows the duration of the years of experience for both the Associates and the Fellows surveyed. The Associates (66) and the Fellows (12) totaled 78 respondents.

\section{Table 2}

Respondents' years of Experience

\begin{tabular}{ccccccc}
\hline $\begin{array}{c}\text { Years of } \\
\text { experience }\end{array}$ & Associates & $\%$ & Fellows & $\%$ & Total & $\%$ \\
\hline $1-5$ & 36 & 100 & - & - & 36 & 46.15 \\
\hline $6-10$ & 19 & 100 & - & & 19 & 24.35 \\
\hline $11-15$ & 7 & & 2 & & 9 & 11.50 \\
\hline $16-20$ & 4 & & 2 & & 6 & 7.68 \\
\hline $21-25$ & - & - & 4 & 100 & 4 & 5.20 \\
\hline $26-30$ & - & - & 2 & 100 & 2 & 2.56 \\
\hline $31-35$ & - & - & 1 & 100 & 1 & 1.28 \\
\hline $36-40$ & - & - & 1 & 100 & 1 & 1.28 \\
\hline Total & 66 & & 12 & & 78 & 100 \\
\hline
\end{tabular}


Source: Author's field survey, 2020.

\subsection{Analysis of Data}

Capital Value $(\mathrm{CV})=$ Site Value $(\mathrm{SV})+$ Building Cost $(\mathrm{New})$ less Building Depreciation

SV $\quad=$ Site Value $=x_{1}$

$\mathrm{BC} \quad=$ Building Cost Anew $=\mathrm{x}_{2}$

Cost Anew $=$ Gross floor $x$ Construction Rate $/ \mathrm{m}^{2}=\mathrm{x}_{3}$

Dep $\quad=$ Depreciation $=\mathrm{x}_{4}$

Table 3

Elements of the cost method used to value property and market value

\begin{tabular}{|c|c|c|c|c|c|}
\hline $\mathrm{S} / \mathrm{N}$ & $\mathrm{X}_{1} \operatorname{Lv}(\mathrm{N})$ & $\begin{array}{l}X_{2} \text { Floor Area } \\
\left(\mathrm{m}^{2}\right)\end{array}$ & $\begin{array}{c}\mathrm{X}_{3} \text { Rate of } \\
\text { Const. } \\
(\mathrm{N} 1,000)\end{array}$ & $\begin{array}{c}\mathrm{X}_{4} \text { Depreciation } \\
\text { rate }(\%)\end{array}$ & $\begin{array}{l}\text { Y Property } \\
\text { value } \\
\text { ( } \$ \text { Million) }\end{array}$ \\
\hline 1 & 3.45 & 110 & 35 & 3 & 7 \\
\hline 2 & 3.2 & 110 & 35 & 25 & 6 \\
\hline 3 & 3.8 & 110 & 40 & 51 & 8 \\
\hline 4 & 3.3 & 110 & 40 & 3 & 7 \\
\hline 5 & 3.5 & 110 & 45 & 8 & 8.1 \\
\hline 6 & 3.5 & 115 & 40 & 6 & 78 \\
\hline 7 & 3.5 & 110 & 40 & 2 & 8 \\
\hline 8 & 3.5 & 110 & 40 & 2 & 7 \\
\hline 9 & 3.2 & 110 & 40 & 15 & 6.5 \\
\hline 10 & 3.7 & 110 & 55 & 15 & 9.5 \\
\hline 11 & 3.5 & 120 & 35 & 20 & 6.8 \\
\hline 12 & 3.3 & 110 & 40 & 20 & 6 \\
\hline 13 & 3.6 & 110 & 35 & 25 & 5.5 \\
\hline 14 & 3.4 & 110 & 35 & 30 & 6.0 \\
\hline 15 & 3.9 & 110 & 45 & 20 & 8 \\
\hline 16 & 3.2 & 115 & 35 & 5 & 7 \\
\hline 17 & 3.5 & 115 & 40 & 2 & 8 \\
\hline 18 & 3.3 & 110 & 35 & 5 & 7 \\
\hline 19 & 2.3 & 110 & 35 & 30 & 6 \\
\hline 20 & 3.5 & 110 & 35 & 6 & .5 \\
\hline 21 & 3.2 & 110 & 35 & 30 & 8 \\
\hline 22 & 3.5 & 115 & 40 & 2 & 8 \\
\hline 23 & 3.4 & 112 & 40 & 3 & 9 \\
\hline 24 & 3.6 & 110 & 35 & 25 & 6 \\
\hline 25 & 3.8 & 110 & 40 & 5 & 8 \\
\hline 26 & 3.1 & 120 & 40 & 15 & 7.2 \\
\hline 27 & 3.6 & 105 & 35 & 10 & 65 \\
\hline 28 & 3.6 & 110 & 40 & 15 & 7.3 \\
\hline 29 & 3.2 & 110 & 40 & 2 & 7.5 \\
\hline 30 & 3.5 & 115 & 40 & 5 & 7.8 \\
\hline 31 & 3.8 & 100 & 35 & 7 & 7.1 \\
\hline 32 & 3.2 & 125 & 40 & 10 & 7.7 \\
\hline 33 & 3.0 & 110 & 45 & 15 & 7.2 \\
\hline 34 & 3.0 & 110 & 35 & 30 & 5.7 \\
\hline 35 & 3.6 & 120 & 40 & 5 & 8.2 \\
\hline 36 & 3.1 & 120 & 40 & 10 & 7.4 \\
\hline 37 & 3.5 & 110 & 40 & 20 & 7.1 \\
\hline 38 & 3.5 & 110 & 40 & 2 & 7.8 \\
\hline 39 & 3.0 & 110 & 40 & 15 & 6.7 \\
\hline 40 & 3.8 & 110 & 35 & 25 & 6.7 \\
\hline 41 & 3.5 & 120 & 35 & 17 & 6.9 \\
\hline 42 & 3.4 & 125 & 45 & 2 & 9.0 \\
\hline 43 & 2.9 & 110 & 45 & 2 & 4.9 \\
\hline 44 & 3.0 & 120 & 50 & 5 & 8.7 \\
\hline
\end{tabular}




\section{$\$$ sciendo}

\begin{tabular}{|c|c|c|c|c|c|}
\hline 45 & 3.1 & 110 & 35 & 1 & 6.5 \\
\hline 46 & 2.6 & 110 & 35 & 20 & 6.0 \\
\hline 47 & 3.0 & 110 & 30 & 3 & 6.2 \\
\hline 48 & 3.0 & 115 & 35 & 5 & 6.8 \\
\hline 49 & 3.5 & 120 & 45 & 1 & 8.9 \\
\hline 50 & 3.5 & 110 & 40 & 12 & 7.3 \\
\hline 51 & 3.7 & 110 & 40 & 1 & 8.1 \\
\hline 52 & 2.9 & 110 & 30 & 25 & 5.4 \\
\hline 53 & 33.5 & 110 & 35 & 12 & 6.8 \\
\hline 54 & 2.7 & 110 & 40 & 15 & 6.4 \\
\hline 55 & 3.0 & 115 & 40 & 8 & 7.2 \\
\hline 56 & 3.5 & 110 & 50 & 9 & 9.0 \\
\hline 57 & 3.3 & 110 & 35 & 30 & 6.0 \\
\hline 58 & 2.6 & 110 & 35 & 25 & 5.9 \\
\hline 59 & 3.2 & 115 & 50 & 12 & 8.3 \\
\hline 60 & 3.5 & 110 & 45 & 7 & 8.1 \\
\hline 61 & 3.5 & 110 & 50 & 2 & 8.9 \\
\hline 62 & 3.5 & 120 & 45 & 15 & 8.0 \\
\hline 63 & 3.7 & 115 & 35 & 3 & 7.6 \\
\hline 64 & 3.9 & 115 & 40 & 13 & 7.9 \\
\hline 65 & 3.5 & 120 & 55 & 5 & 9.8 \\
\hline 66 & 3.5 & 115 & 45 & 20 & 7.6 \\
\hline 67 & 3.0 & 120 & 45 & 15 & 7.6 \\
\hline 68 & 3.0 & 110 & 40 & 8 & 7.0 \\
\hline 69 & 3.5 & 110 & 35 & 17 & 6.6 \\
\hline 70 & 3.5 & 110 & 40 & 9 & 7.5 \\
\hline 71 & 3.5 & 110 & 45 & 25 & 7.6 \\
\hline 72 & 3.5 & 120 & 50 & 5 & 9.2 \\
\hline 73 & 3.6 & 125 & 50 & 13 & 9.1 \\
\hline 74 & 3.7 & 110 & 35 & 15 & 6.9 \\
\hline 75 & 3.8 & 115 & 35 & 8 & 7.5 \\
\hline 76 & 3.8 & 115 & 45 & 25 & 7.7 \\
\hline 77 & 3.9 & 115 & 45 & 5 & 8.8 \\
\hline \multirow[t]{2}{*}{78} & 4.0 & 125 & 50 & 5 & 9.9 \\
\hline & $\begin{aligned} \sum X_{1} & =263.95 \\
X_{1} & =3384\end{aligned}$ & $\underset{X_{2}}{\sum X_{2}=8582}$ & $\begin{array}{l}\sum \mathrm{X}_{3}=3055 \\
\mathrm{X}_{3}=39167\end{array}$ & $\underline{X}_{X_{4}}=11346$ & $\begin{array}{l}\frac{\sum Y}{Y}=575.4 \\
Y=7374\end{array}$ \\
\hline
\end{tabular}

Source: Author's field survey, 2020.

Table 3 shows a presentation of the values of elements applied by Estate Surveyors and valuers in the valuation of the same property; the table shows the range in site values to be between $2.3-4.0$ million naira, the range of values of the floor area to fall between $100-125$ square meters, rates of construction applied by Estate Surveyors and valuers to value this property to range from 35 - 55 thousand naira, while the depreciation rates applied by the Estate Surveyors and valuers - from $1-30 \%$.

Table 4

Analysis of variance of Elements of the Applied Cost Method

\begin{tabular}{lllllllllll}
\hline $\mathrm{S} / \mathrm{N}$ & $\mathrm{X}_{1}-\mathrm{X}^{1}$ & $\left(\mathrm{X}_{1}-\mathrm{X}_{1}\right)^{2}$ & $\mathrm{X}_{2}-\mathrm{X}_{2}$ & $\left(\mathrm{X}_{2}-\mathrm{X}_{2}\right)^{2}$ & $\mathrm{X}_{3}-\mathrm{X}_{3}$ & $\left(\mathrm{X}_{3}-\mathrm{X}_{3}\right)^{2}$ & $\mathrm{X}_{4}-\mathrm{X}_{4}$ & $\left(\mathrm{X}_{4}-\mathrm{X}_{4}\right)^{2}$ & $\mathrm{Y}-\mathrm{Y}$ & $\mathrm{Y}-\mathrm{Y}) 2$ \\
\hline $\mathbf{1}$ & 0.066 & 0.004 & -0.025 & 0.001 & -4.167 & 17.364 & -3.346 & 11.196 & -0.374 & 0.095 \\
\hline $\mathbf{2}$ & 0.184 & 0.034 & -0.025 & 0.001 & -4.167 & 17.364 & 13.654 & 186.432 & -0.374 & 1.888 \\
\hline $\mathbf{3}$ & 0.416 & 0.173 & -0.025 & 0.001 & 0.833 & 0.694 & -6.341 & 40.208 & 0.626 & 0.392 \\
\hline $\mathbf{4}$ & 0.316 & 0.010 & -0.025 & 0.001 & 0.833 & 0.694 & -8.346 & 69.656 & -0.374 & 0.095 \\
\hline $\mathbf{5}$ & 0.116 & 0.014 & -0.025 & 0.001 & 5.833 & 34.023 & -3.346 & 11.196 & 0.726 & 0.527 \\
\hline $\mathbf{6}$ & 0.116 & 0.014 & 4.975 & 24.75 & 0.833 & 0.694 & -5.346 & 28.580 & 0.426 & 0.182 \\
\hline $\mathbf{7}$ & 0.116 & 0.014 & -0.025 & 0.001 & 0.833 & 0.694 & 9.346 & 87.348 & 0.626 & 0.392 \\
\hline $\mathbf{8}$ & 0.116 & 0.014 & -0.025 & 0.001 & 0.833 & 0.694 & 9.346 & 87.348 & -0.374 & 0.095 \\
\hline $\mathbf{9}$ & 0.184 & 0.034 & -0.025 & 0.001 & 0.833 & 0.694 & 3.654 & 13.352 & -0.874 & 0.764 \\
\hline $\mathbf{1 0}$ & 0.316 & 0.010 & -0.025 & 0.001 & 15.833 & 50.684 & -6.346 & 40.208 & -2.126 & 4.520 \\
\hline
\end{tabular}




\begin{tabular}{|c|c|c|c|c|c|c|c|c|c|c|}
\hline 11 & 0.116 & 0.014 & 9.975 & 99.50 & -4.167 & 17.364 & 8.654 & 74.892 & -0.574 & 0.329 \\
\hline 12 & 0.084 & 0.007 & -0.025 & 0.001 & 0.833 & 0.694 & 8.654 & 74.892 & -1.374 & 1.888 \\
\hline 13 & 0.216 & 0.047 & -0.025 & 0.001 & -4.167 & 17.364 & 13.654 & 186.432 & -1.874 & 3.512 \\
\hline 14 & 0.016 & 0.000 & -0.025 & 0.001 & -4.167 & 17.364 & 18.654 & 347.972 & -1.374 & 1.888 \\
\hline 15 & 0.516 & 0.266 & -0.025 & 0.001 & 5.833 & 34.023 & 3.654 & 13.352 & -0.626 & 0.392 \\
\hline 16 & 0.184 & 0.034 & 4.975 & 24.75 & -4.167 & 17.364 & -6.346 & 40.208 & -0.374 & 0.095 \\
\hline 17 & 0.316 & 0.010 & 4.975 & 24.75 & 0.833 & 0.694 & 9.346 & 87.348 & -0.626 & 0.392 \\
\hline 18 & 0.084 & 0.007 & -0.025 & 0.001 & -4.167 & 17.364 & -6.346 & 40.208 & -0.374 & 0.095 \\
\hline 19 & 1.084 & 1.084 & -0.025 & 0.001 & -4.167 & 17.364 & 18.654 & 347.972 & -1.374 & 1.888 \\
\hline 20 & 0.116 & 0.014 & -0.025 & 0.001 & -4.167 & 17.364 & 5.346 & 28.580 & -1.874 & 3.512 \\
\hline 21 & 0.184 & 0.034 & -0.025 & 0.001 & -4.167 & 17.364 & 18.654 & 347.972 & -0.626 & 0.392 \\
\hline 22 & 0.116 & 0.014 & 4.975 & 24.75 & 0.833 & 0.694 & 9.346 & 87.348 & -0.626 & 0.392 \\
\hline 23 & 0.016 & 0.000 & 1.975 & 3.901 & 0.833 & 0.694 & -8.346 & 69.656 & -1.626 & 2.644 \\
\hline 24 & 0.216 & 0.047 & -0.025 & 0.001 & -4.167 & 17.364 & 13.654 & 186.432 & -1.374 & 1.888 \\
\hline 25 & 0.416 & 0.173 & -0.025 & 0.001 & 0.833 & 0.694 & -6.346 & 40.208 & -0.626 & 0.392 \\
\hline 26 & 0.284 & 0.081 & 9.975 & 99.50 & 0.833 & 0.694 & 3.654 & 13.352 & -0.174 & 0.030 \\
\hline 27 & 0.216 & 0.047 & -5.25 & 25.25 & -4.167 & 17.364 & -1.346 & 1.812 & -0.874 & 0.764 \\
\hline 28 & 0.216 & 0.047 & -0.025 & 0.001 & 0.833 & 0.694 & 3.654 & 13.352 & -0.074 & 0.006 \\
\hline 29 & 0.184 & 0.034 & -0.025 & 0.001 & 0.833 & 0.694 & 9.346 & 87.348 & -0.126 & 0.016 \\
\hline 30 & 0.116 & 0.014 & 4.975 & 24.75 & 0.833 & 0.694 & -6.346 & 40.208 & -0.426 & 0.182 \\
\hline 31 & 0.416 & 0.173 & -10.025 & 100.50 & -4.167 & 17.364 & -4.346 & 18.888 & -0.274 & 0.075 \\
\hline 32 & 0.184 & 0.034 & 14.975 & 24.75 & 0.833 & 0.694 & -1.346 & 1.812 & -0.326 & 0.106 \\
\hline 33 & 3.384 & 11.452 & -0.025 & 0.001 & 0.833 & 0.694 & 3.654 & 13.352 & -0.174 & 0.034 \\
\hline 34 & 3.384 & 11.452 & -0.025 & 0.001 & -4.167 & 17.364 & 18.654 & 347.972 & -1.674 & 2.802 \\
\hline 35 & 0.216 & 0.047 & 9.975 & 99.50 & 0.833 & 0.694 & -6.346 & 40.208 & -0.826 & 0.682 \\
\hline 36 & 0.284 & 0.081 & 9.975 & 99.50 & 0.833 & 0.694 & -1.346 & 1.812 & -0.026 & 0.001 \\
\hline 37 & 0.116 & 0.014 & -0.025 & 0.001 & 0.833 & 0.694 & 8.654 & 743892 & -0.274 & 0.075 \\
\hline 38 & 0.116 & 0.014 & -0.025 & 0.001 & 0.833 & 0.694 & 9.346 & 87.348 & -0.426 & 0.182 \\
\hline 39 & 3.384 & 11.452 & -0.025 & 0.001 & 0.833 & 0.694 & 3.654 & 13.352 & -0.674 & 0.454 \\
\hline 40 & 0.416 & 0.416 & -0.025 & 0.001 & -4.167 & 17.364 & 13.654 & 186.432 & -0.674 & 0.454 \\
\hline 41 & 0.014 & 0.116 & 9.975 & 99.50 & -4.167 & 17.364 & 5.654 & 31.968 & -0.474 & 0.225 \\
\hline 42 & 0.000 & 0.116 & 14.975 & 224.25 & 5.833 & 34.023 & 9.346 & 87.348 & 1.626 & 2.644 \\
\hline 43 & 0.193 & -0.439 & -0.025 & 0.001 & 5.833 & 34.023 & 9.346 & 87.348 & -2.474 & 6.12 \\
\hline 44 & 11.452 & 3.384 & 9.975 & 99.50 & 10.833 & 17.364 & -6.346 & 40.208 & 1.326 & 1.758 \\
\hline 45 & 0.081 & -0.284 & -0.025 & 0.001 & -4.167 & 17.364 & -10.346 & 107.040 & -0.874 & 0.764 \\
\hline 46 & 0.615 & -0.784 & -0.025 & 0.001 & -4.167 & 17.364 & 8.654 & 74.892 & -1.374 & 1.888 \\
\hline 47 & 11.452 & 3.384 & -0.025 & 0.001 & -9.167 & 84.034 & 8.346 & 69.656 & -1.174 & 1.378 \\
\hline 48 & 11.452 & 3.384 & 4.975 & 24.75 & -4.167 & 17.364 & 6.346 & 40.208 & -0.574 & 0.329 \\
\hline 49 & 0.014 & 0.116 & 9.975 & 99.50 & 5.833 & 34.023 & 10.346 & 107.040 & 1.526 & 2.329 \\
\hline 50 & 0.014 & 0.116 & -0.025 & 0.001 & 0.833 & 0.694 & 0.654 & 0.428 & -0.074 & 0.006 \\
\hline 51 & 0.010 & 0.316 & -0.025 & 0.001 & 0.833 & 0.694 & 10.346 & 107.040 & 0.726 & 0.527 \\
\hline 52 & 0.234 & -0.484 & -0.025 & 0.001 & -9.167 & 84.034 & 13.654 & 186.432 & -1.974 & 3.897 \\
\hline 53 & 0.014 & 0.116 & -0.025 & 0.001 & -4.167 & 17.364 & 0.654 & 0.428 & -0.574 & 0.329 \\
\hline 54 & 0.468 & -0.684 & -0.025 & 0.001 & 0.833 & 0.694 & 3.654 & 13.352 & -0.974 & 0.899 \\
\hline 55 & 11.452 & 3.384 & 4.975 & 24.75 & 0.833 & 0.694 & -3.346 & 11.196 & -0.174 & 0.034 \\
\hline 56 & 0.014 & 0.116 & 9.975 & 99.50 & 10.833 & 117.364 & 2.346 & 5.504 & 1.626 & 2.644 \\
\hline 57 & 0.007 & 0.084 & -0.025 & 0.001 & -4.167 & 17.364 & 18.654 & 347.972 & -1.374 & 1.888 \\
\hline 58 & 0.614 & 0.784 & -0.025 & 0.001 & 0.833 & 0.694 & 13.654 & 186.432 & -1.474 & 2.173 \\
\hline 59 & 0.034 & 0.184 & 4.975 & 24.75 & 10.833 & 117.364 & 0.654 & 0.428 & 0.926 & 0.858 \\
\hline 60 & 0.014 & 0.116 & -0.025 & 0.001 & 5.833 & 34.023 & 4.346 & 18.888 & 0.726 & 0.527 \\
\hline 61 & 0.014 & 0.116 & -0.025 & 0.001 & 10.833 & 117.364 & 9.346 & 87.348 & 1.526 & 2.329 \\
\hline 62 & 0.014 & 0.116 & 9.975 & 99.50 & 5.833 & 34.023 & 3.654 & 13.352 & 0.626 & 0.392 \\
\hline 63 & 0.010 & 0.316 & 4.975 & 24.75 & -4.167 & 17.364 & 8.346 & 69.656 & 0.256 & 0.066 \\
\hline 64 & 0.266 & 0.516 & 4.975 & 24.75 & 0.833 & 0.694 & 1.654 & 2.736 & 0.526 & 0.277 \\
\hline 65 & 0.014 & 0.116 & -9.975 & 99.50 & 5.833 & 250.684 & -6.346 & 40.208 & 2.426 & 5.885 \\
\hline 66 & 0.014 & 0.116 & 4.975 & 24.75 & 5.833 & 34.023 & -9.346 & 87.348 & 0.256 & 0.066 \\
\hline 67 & 11.452 & 3.384 & -9.975 & 99.50 & 5.833 & 34.023 & 3.654 & 13.352 & 0.256 & 0.066 \\
\hline 68 & 11.452 & 3.384 & 0.025 & 0.001 & 0.833 & 0.694 & 3.346 & 11.196 & -0.374 & 0.095 \\
\hline 69 & 0.014 & 0.116 & 0.025 & 0.001 & -4.167 & 17.364 & 5.654 & 31.968 & -0.774 & 0.599 \\
\hline
\end{tabular}




\section{$\$$ sciendo}

\begin{tabular}{|c|c|c|c|c|c|c|c|c|c|c|}
\hline 70 & 0.014 & 0.116 & 0.025 & 0.001 & 0.833 & 0.694 & -2.346 & 5.504 & 0.126 & 0.016 \\
\hline 71 & 0.014 & 0.116 & -9.975 & 99.50 & 5.833 & 34.023 & 13.654 & 186.432 & 0.256 & 0.066 \\
\hline 72 & 0.014 & 0.116 & -9.975 & 99.50 & 10.833 & 117.364 & -6.346 & 40.208 & 1.826 & 3.334 \\
\hline 73 & 0.047 & 0.216 & 14.975 & 224.25 & 10.833 & 17.364 & 1.654 & 2.736 & 1.726 & 2.979 \\
\hline 74 & 0.010 & 0.316 & 0.025 & 0.001 & -4.167 & 17.364 & 3.654 & 13.352 & -0.474 & 0.225 \\
\hline 75 & 0.173 & 0.416 & -4.975 & 24.75 & -4.167 & 17.364 & 3.346 & 11.196 & 0.125 & 0.016 \\
\hline 76 & 0.173 & 0.416 & -4.975 & 24.75 & 5.833 & 34.023 & 13.654 & 186.432 & 0.326 & 0.106 \\
\hline 77 & 0.266 & 0.516 & -4.975 & 24.75 & 5.833 & 34.023 & -6.346 & 40.208 & 1.426 & 2.034 \\
\hline \multirow[t]{2}{*}{78} & 0.380 & 0.616 & -4.975 & 24.75 & 10.833 & 117.364 & -6.346 & 40.208 & 2.526 & 6.381 \\
\hline & & $\begin{array}{l}\sum(X-X)^{2}= \\
159.775\end{array}$ & & $\begin{array}{l}\sum\left(\mathrm{X}_{2-} \mathrm{X}_{2}\right)^{2}= \\
2,262.695\end{array}$ & & $\begin{array}{l}\sum_{2,354.103}\left(X_{3}-X_{3}=\right. \\
2,103\end{array}$ & & $\begin{array}{l}\sum\left(\mathrm{X}_{4}-\mathrm{X}_{4}\right) \\
=6005.854\end{array}$ & & $\begin{array}{l}\sum(y-y) 2 \\
=88.368\end{array}$ \\
\hline
\end{tabular}

Source: Authors' computation, 2020.

Table 4 shows the analysis of total dispersion from the mean of the elements of cost applied in the cost method of valuation. The analysis of dispersion revealed that: variable $\mathrm{x}_{1}$ (land value) shows a dispersion of 159.775 , variable $\mathrm{x}_{2}$ (floor area) shows a dispersion of 2262.695, variable $\mathrm{x}_{3}$ (rate of construction) shows a dispersion of 2354.103, variable $\mathrm{x}_{4}$ (rate of depreciation) shows a dispersion of 6005.854 , and y shows a dispersion of 88.368.

4.2. Analysis of variance showing standard deviation and the coefficient of variation (CV) of a variable as used by the estate surveyors for valuation

Table 5

Summary of the analysis showing measures of disparity of values of variables adopted in the valuation

\begin{tabular}{lllllll}
\hline $\mathrm{S} / \mathrm{N}$ & Variables & $\Sigma(\mathrm{x})$ & Mean & $\Sigma\left(\mathrm{x}-\mathrm{x}^{2} / \mathrm{N}\right.$ & $\sqrt{ }\left(\mathrm{x}-\mathrm{x}^{2} / \mathrm{N}\right.$ & $\mathrm{CV}$ \\
\hline 1 & Site value & $\Sigma \mathrm{x} 1=263.95$ & $\mathrm{X} 1=3.384$ & 2.048 & 1.431 & 0.423 \\
\hline 2 & $\begin{array}{l}\text { construction } \\
\text { cost } / \mathrm{m}^{2}\end{array}$ & $\Sigma \mathrm{x} 2=8582$ & $\mathrm{X} 2=110.025$ & 29.009 & 5.386 & 0.049 \\
\hline 3 & Gross Floor Area & $\Sigma \times 3=3055$ & $\mathrm{X} 3=39.167$ & 30.181 & 5.494 & 0.138 \\
\hline 4 & Depreciation rates & $\Sigma \mathrm{x} 4=885$ & $\mathrm{X} 4=11.346$ & 76.998 & 8.775 & 0.773 \\
\hline 5 & Property Value & $\Sigma \mathrm{y}=575.4$ & $\mathrm{Y}=7.374$ & 1.136 & 1.066 & 0.145 \\
\hline
\end{tabular}

Source: Authors computation, 2020.

Table 5 is a summary of the mean values, variance, standard deviation and coefficient of variation for each variable applied by the Estate Surveyors and Valuers for the property under study. The means for the variables are $X_{1}=3.384, X_{2}=110.025, X_{3}=39.167 \mathrm{and} X_{4}=11.346$, whereas variable $\mathrm{Y}$ has a mean of 7.374 . Variable $\mathrm{x}_{1}$ (Site value) shows a variance (mean dispersion) of 2.048 and $\sigma$ of 1.431 . The variable $\left(\mathrm{x}_{2}\right)$ of floor area is characterized by a variance of 29.009 and standard deviation $(\sigma)$ of 5.386. Variable $\mathrm{x}_{3}$ (rate of construction) shows a variance (mean dispersion) of $=30.181$ and $\sigma$ of 5.494. Variable $x_{4}$ (the rate of depreciation), shows a variance (mean dispersion) of 76.998 and $\sigma$ of 8.775 , while variable y (property value) shows a variance (mean dispersion) of 1.066 and $\sigma$ of 1.066 . The coefficients of variation of the variables are $\mathrm{X} 1=0.423, \mathrm{X}_{2}=0.049, \mathrm{X}_{3}=0.138, \mathrm{X}_{4}=0.773$ and $\mathrm{Y}=0.145$. A higher coefficient of variation indicates a higher variability, while a low coefficient of variation indicates a low variability. Thus, the Depreciation rate $\left(\mathrm{X}_{4}\right)$ has the highest variability, followed by Site value and Gross Floor Area.

\subsection{Test of significance to the variance}

Ho: The contribution of variables to property value variance in Estate Surveyors' and valuers' application of the cost method of valuation is not significant. The F ratio between the variables is thus calculated as:

$$
\begin{gathered}
\mathrm{F}=\frac{S_{1}^{2}}{S_{2}^{2}} \text {, where } S_{1}^{2}=\frac{\sum x_{1}-\overline{\bar{x}_{1}}}{n_{1}-1} \text { and } S_{2}^{2}=\frac{\sum x_{2}-\overline{\bar{X}_{2}}}{n_{2}-1} \\
F_{1}=\frac{2.048}{1.136}=1.8 ; F_{2}=\frac{29.01}{1.136}=25.54 ; F_{3}=\frac{30.18}{1.136}=26.57 ; F_{4}=\frac{77}{1.136}=67.78
\end{gathered}
$$


Degrees of freedom for $x_{1}, x_{2}, x_{3}, x_{4}=\left(n_{2}-1\right)=78-1=77 ; v_{1}, v_{2}, v_{3}$ and $v_{4}=77$

The computation of the $\mathrm{F}$ ratio at $5 \%$ level of significance for $\mathrm{v}_{1}, \mathrm{v}_{2}, \mathrm{v}_{3} \mathrm{v}_{4}$ and $\mathrm{v}_{5}=77$. Since the calculated values of $\mathrm{F}_{1}=1.8, \mathrm{~F}_{2}=25.54 \mathrm{~F}_{3}=26.57$ and $\mathrm{F}_{4}=67.78$, which are higher than the table value of 1.3, the F ratios for all variables were therefore significant at a $5 \%$ level of error and, as such, we reject the null hypothesis and conclude that the variables contributing to property value variance are significant.

\section{Discussion and Conclusion}

The analyses of variance show that: variable $x_{1}$ (site value) displays a variance of 0.052 and standard deviation of 0.227 . The variable (floor area, $x_{2}$ ) shows a variance of 6.004 and standard deviation of 2.4503. The variable (rate of construction, $x_{3}$ ) shows a variance of 6.784 and standard deviation of 2.6046, which is also fairly significant; variable $\mathrm{x}_{4}$ (rate of depreciation) shows a variance of 113.974 and Standard deviation of 10.6758. This means that the rate of depreciation is the variable that contributes most to valuation variance in the cost method of valuation. This might be attributed to the variance in valuers' sense of judgment connected with their years of experience.

The analysis of the variables contributing to the total valuation variance in the application of the cost method of valuation shows that land values contribute $1.422 \%$, floor area contributes $15.4 \%$, the rate of construction applied contributes $16.3 \%$ and depreciation rate measured by valuers contributes $66.9 \%$ to the total variance in market value when the cost method is used. This is attributable to the use of the estimated percentage or rule of thumb method applied by most Estate Surveyors and Valuers in Uyo, in which they rely on their varied sense of judgment and years of experience, with most valuers unfortunately having have only a few years of experience, hence resulting in the variance in depreciation estimates.

Considering the calculated $\mathrm{F}$ values of $115.46,130.46,2191.8,1.13,18.983$ and 16.80, compared to a table value of 2.66, the test of hypothesis was conducted, with the null hypothesis being rejected and the alternative hypothesis accepted. Therefore, the variables contributing to total valuation variance were significant for land value and the subjective measurements of valuation variables, especially depreciation, the rate of construction and linear measurements. These were determinants of valuation variance.

The study observed that the incorrect application of depreciation rate during computation contributed to the variance in the cost method of valuation. The other sources of errors observed were the application of the building construction rate $/ \mathrm{m}^{2}$ and the errors due to linear measurements of the buildings. The consequences of these anomalies could cause clients to receive lower or higher values for their asset. The study reveals that depreciation rates applied by different valuers account for over $60 \%$ of total variance in values among estate surveyors and valuers, while construction rates and the linear measurements of real properties were slightly significant. The errors in linear measurements usually occur during the measurements of irregularly-shaped real properties.

The research recommends that Estate surveyors and Valuers applying the cost method should adopt a regional-based linear or non-linear depreciation model that best suits the region's real estate framework and depreciation approach. The variance coming from the measurement and computation of areas and volumes can be controlled by using tools, such as the electronic distance measuring equipment (planimeter) with high precision. Automated instruments and packages, such as GPRS, goggle earth pro, Microsoft excel, among other valuation software should be adopted. The rates to be applied for construction and statutory use should be published on the institution's website and made available to valuers.

\section{References}

Adair, A., Berry, J., \& McGreal, S. (1996). Valuation of Residential property: Analysis of participant Behaviour. Journal of Property Valuation and Investment, 14(1), 20-35. https://doi.org/10.1108/14635789610107453 


\section{S sciendo}

Adegoke, O. J. (2016). Effects of valuation variance and inaccuracy on Nigerian commercial property market. Journal of Property Investment \& Finance, 34(3), 276-292. https://doi.org/10.1108/JPIF-08$\underline{2014-0056}$

Ayedun, C. A., Oloyede, S. A., \& Durodola, O. D. (2012). Empirical study of the causes of valuation variance and inaccuracy in Nigeria. International Business Research, 5(3), 71-79. https://doi.org/10.5539/ibr.v5n3p71

Bello, I. K. (2014). Depreciation measurement in cost method of valuation in Lagos metropolis. American Journal of Social and Management Sciences. www.scihub.org/Ajsms. Retrieved 22/03/2018.

Bello, I. K., Ogunba, O. A., \& Adegunle, T. O. (2015). Appropriateness of depreciation measurement in the cost method of property valuation in Lagos, Nigeria. Journal of Business Management and Economic 6(2). 017-026. http:// www.e3journals.org

Effiong, J. B. (2015). A Comparative Study of Valuation Variance and Accuracy between Nigeria and UK. International Letters of Social and Humanistic Sciences, 57, 94-105. https://doi.org/10.18052/www.scipress.com/ILSHS.57.94

Görzig, B. (2007) Depreciation in EU Member States: Empirical and Methodological Differences. Helsinki workshop of the EU KLEMS project; Berlin.

Guidance Note, R. I. C. S. (2018). Depreciated Replacement Cost Method for Financial Reporting (First Ed.). Royal Institution of Chartered Surveyors, London. https://www.rics.org/globalassets/ricswebsite/media/upholding-professional-standards/sector-standards/valuation/drc-method-ofvaluation-for-financial-reporting-1 ${ }^{\text {st }}$ edition-rics.pdf: Retrieved November 13, 2020

Hutchison, N., Macgregor, B., \& Nanthakumaran, N. (1995). Variations in the Capital Valuations of UK Commercial Property: Do Valuers Get It Right? RICS 1995 Cutting Edge Conference Aberdeen, September.

International Valuation Standards (IVSC). (2013). Valuation Information paper Iv-The cost Approach for financial Reporting. International Valuation Standard Commission.

International Valuation Standards (IVSC). (2019). Framework and Requirements. International Valuation Standards Council.

Kalan, O. (2018). Application of Regional Specific Depreciation Formulars in highway Capital Stocks: Evidence From New York and New Jersey. Paper accepted for Presentation in the Transportation Research Board's 98th Annual Meeting, Washington, D.C., 2019.

Kalu, I. U. (2001). Property Valuation and Appraisal. Bon Publication.

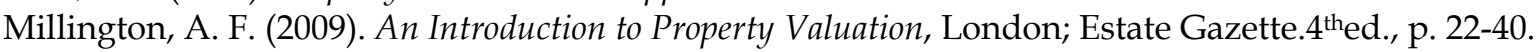

NIESV DIRECTORY. (2018). The Nigerian Institution of Estate Surveyors and Valuers Directory. http:// firms.niesv.org.ng/niesva-zfirms.php. Retrieved on March 19, 2020.

Parker, D. (1998).Valuation accuracy: An Australian Perspective. Paper presented at the $4^{\text {th }}$ Pacific Rim Real Estate Society Conference, Perth. http://www.prres.net/proceedings: Retrieved February 23, 2020.

Plimmer, F., \& Sayce, S. (2006). Depreciated Replacement Cost - Consistent Methodology? https://www.fig.net/resources/proceedings/fig_proceedings/fig2006/papers/ts86/ts86_01_pli mmer_sayce_0268.pdf. Retrieved: March 19, 2020.

Roca Cladera, J. (1996). La valoracionImmobiliaria: Ciencia, arte U Oficio? In CT/Catastro. Director General Office-Ministry of Economic Development.

Royal Institution of Chartered Surveyors (RICS). (2019). RICS Valuation - Global Standards 2017: UK national supplement Issued November 2018. Published by the Royal Institution of Chartered Surveyors (RICS) Parliament Square London SW1P 3AD www.rics.org Valuation Professional Group Taqueem Academy (2016). Saudi Authority for Accredited Valuers Manual: Valuation/Cost Approach. http//:www.taqeem.gov.sa. Retrieved 10 February, 2020.

The Green Book. (2019). The Nigeria Valuation Standards. Estate Surveyors and Valuers Registration Board of Nigeria.

Vaz, J. F. (2015). Real Estate Appraisal and Subjectivity. European Scientific Journal, 20(1), 55-66.

Yeboah \& Ayitey. (2006). Assessing Depreciation for Valuation Purposes - A Decompositional Approach. Promoting Land Administration and Good Governance 5th FIG Regional Conference held in Accra, Ghana, March 8-11, 2006. 